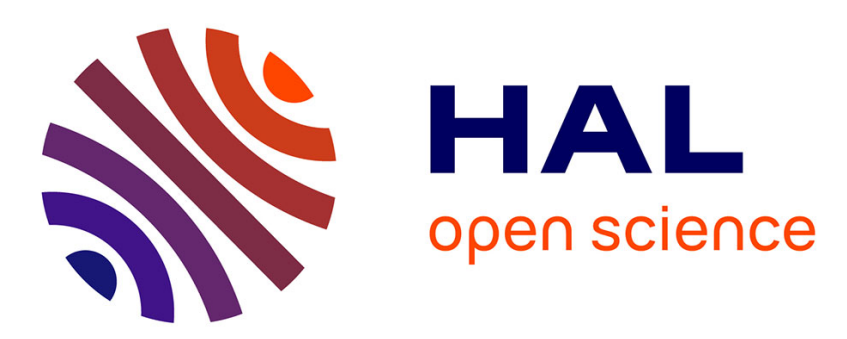

\title{
Shared destinies and the measurement of social risk equity
}

Thibault Gajdos, John Weymark, Claudio Zoli

\section{To cite this version:}

Thibault Gajdos, John Weymark, Claudio Zoli. Shared destinies and the measurement of social risk equity. 2008. halshs-00344468

\section{HAL Id: halshs-00344468 \\ https://shs.hal.science/halshs-00344468}

Submitted on 4 Dec 2008

HAL is a multi-disciplinary open access archive for the deposit and dissemination of scientific research documents, whether they are published or not. The documents may come from teaching and research institutions in France or abroad, or from public or private research centers.
L'archive ouverte pluridisciplinaire HAL, est destinée au dépôt et à la diffusion de documents scientifiques de niveau recherche, publiés ou non, émanant des établissements d'enseignement et de recherche français ou étrangers, des laboratoires publics ou privés. 


\section{Documents de Travail du Centre d'Economie de la Sorbonne}

C

E

$\mathrm{S}$

W

0

1

k

i

n

g

$\mathrm{P}$

a

$\mathrm{p}$

e

1

$\mathrm{S}$

CENTRE NATIONAL

DE LA RECHERCHE

SCIENTIFIQUE

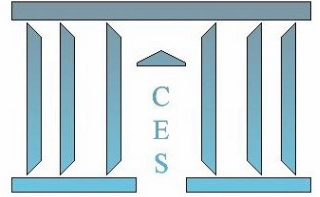

Shared Destinies and the Measurement

of Social Risk Equity

Thibault GAJDOS, John A. WEYMARK, Claudio ZOLI

2008.69 


\section{Shared Destinies and the Measurement of Social Risk Equity \\ by}

Thibault Gajdos, John A. Weymark, and Claudio Zoli

October 2008

Thibault Gajdos, CNRS-Centre d'Economie de la Sorbonne, Université Paris 1 (Panthéon-Sorbonne), Maison des Sciences Economiques, 106-112 boulevard de L'Hôpital, 75647 Paris Cedex 13, FRANCE.

(e-mail: thibault.gajdos@univ-paris1.fr)

John A. Weymark, Department of Economics, Vanderbilt University, VU Station B \#35189, 2301 Vanderbilt Place, Nashville, TN 37235-1819, U.S.A. (e-mail: john.weymark@vanderbilt.edu)

Claudio Zoli, Dipartimento di Scienze Economiche, Università degli Studi di Verona, Polo "G. Zanotto", Viale dell'Università 4, 37129 Verona, ITALY. (e-mail: claudio.zoli@univr.it) 


\title{
Shared Destinies and the Measurement of Social Risk Equity
}

\author{
by Thibault Gajdos, John A. Weymark, and Claudio Zoli
}

\begin{abstract}
The evaluation of social risk equity for alternative probability distributions over the potential sets of fatalities is analyzed axiomatically. Fishburn and Straffin [Equity considerations in public risks valuation, Operatons Research 37 (1999), 229-239] have identified a necessary and sufficient condition for two social risk distributions to be judged to be socially indifferent whenever their associated distributions of risk of death for individuals and for the number of fatalities are the same. It is argued that this approach does not permit society to exhibit any concern for the number of people an individual perishes with. A weakening of the Fishburn-Straffin condition that is compatible with a concern for shared destinies is proposed.
\end{abstract}

Keywords and Phrases: social risk evaluation, social risk equity, public risk, shared destinies

Résumé. Nous proposons une analyse axiomatique de l'évaluation de l'exposition collective à un risque de décès. Il s'agit plus précisément de comparer, en termes d'équité, différentes distributions de probabilité de décès pour l'ensemble des sous-groupes de la population. Fishburn and Straffin [Equity considerations in public risks valuation, Operatons Research 37 (1999), 229-239] ont identifié une condition nécessaire et suffisante pour que deux risques sociaux soient jugés également équitables dès lors qu'ils conduisent d'une part à des probabilités individuelles de décès identiques, et d'autre part aux même distributions de probabilité sur le nombre de décès. Nous montrons qu'une telle approche ne permet pas de prendre en compte l'importance que peut avoir le fait de partager son destin avec un plus ou moins grand nombre d'autres personnes. Nous proposons un affaiblissement de la condition de Fishburn et Straffin qui permet de tenir compte de l'influence de la communauté de destin dans l'évaluation d'un risque social.

Mots clés: évaluation du risque social, équité sociale face au risque, risque collectif, communauté de destin.

Journal of Economic Literature Classification Numbers: D63, D81, H43 


\section{Introduction}

Societies face risks and design public policies to manage these risks. In many cases, these risks involve the potential loss of life. Examples of such risks include hurricanes, earthquakes, epidemics, terrorist attacks, nuclear disasters, and the collapse of bridges. In order to decide which amomg the feasible riskreducing policies to implement, it is necessary to evaluate these risks from a social point of view. For risks of death, common ways of evaluating the success of a policy are to compute the expected number of lives saved or the reduction in the average risk of death. Such measures fail to account for some important dimensions of risk evaluation, such as the equity of the resulting distribution of risks or social risk attitudes about the potential number of fatalities. For example, the construction of a levee that ensures that one neighbourhood in a city is safe from floods while leaving the rest of the city unprotected may be regarded as being socially inferior to the construction of a more extensive system of levees that reduces, but does not eliminate, the risk of flooding in all parts of the city. Society may also prefer to have a nonnuclear military defence strategy rather than to acquire nuclear weapons, even if the expected loss of life is the same in both cases, so as to avoid the possibility of a catastrophic loss of life with the latter strategy.

Beginning with Keeney (1980a,b,c), an extensive theoretical literature has developed that is devoted to the analysis of social risk equity. See, for example, Broome (1982), Fishburn (1984), Fishburn and Sarin (1991), Fishburn and Straffin (1989), Harvey (1985), Keeney and Winkler (1985), and Sarin (1985). Attitudes towards social risk equity have also been investigated using surveys and interviews by Keller and Sarin (1988) and Bian and Keller (1999).

One issue that has been considered in this literature concerns the extent to which a satisfactory index of overall social risk equity can be obtained by aggregating indices that measure different dimensions of social risk. ${ }^{1}$ Suppose that we are concerned with the risk of death. Each subgroup of the population is a potential fatality set; i.e., each subgroup could be the set of individuals who perish as a result of their exposure to the risk being considered. A social risk distribution is a probability distribution over these fatality sets. Given such a probability distribution, one can compute each

\footnotetext{
${ }^{1}$ For a thoughtful recent discussion of some of the other issues that arise when evaluating social risks, see Fleurbaey (2006).
} 
individual's probabilty of death and the probability that there will be any particular number of fatalities. Using this information, Keeney and Winkler (1985), Sarin (1985), and Fishburn and Sarin (1991) have constructed indices of social risk equity for the distributions of individual likelihoods of death and indices of social risk equity for the distributions on the number of fatalities which are then aggregated into an overall index of social risk equity. ${ }^{2}$ This two-stage procedure would be justified if two social risk distributions are judged to be socially indifferent whenever their associated distributions of risk for individuals and for the number of fatalities are the same. Assuming that the overall index is continuous, Fishburn and Straffin (1989) have identified a necessary and sufficient condition for this to be the case.

In this article, we argue that this approach fails to take account of an important dimension of social risk equity. Individuals care about the number of people who share their destiny. It is quite a different matter to die alone than to perish with a number of other individuals. As we shall show, it is possible for the distributions over how many other people one dies with to differ between two social risk distributions even though they share the same distributions of risk of death for individuals and the same distributions of risk for the number of fatalities. It is not possible for society to exhibit any concern for this "shared destinies" aspect of social risk equity if one adopts Fishburn and Straffin's condition. We propose a weakening of their condition that permits such a concern and show that our condition implies that the social risk evaluation only depends on the probabilities that an individual dies with $k$ other individuals for all possible values of $k$.

In Section 2, we present our formal model of social risk equity evaluation. We consider some examples that illustrate the importance of the three dimensions of social risk equity that we have identified in Section 3. In Section 4, after first discussing the Fishburn-Straffin condition, we show how this condition can be weakened so as to be compatible with a social concern for shared destinies and we identify the restrictions on the social risk evaluation implied by our axioms. We offer some concluding remarks in Section 5.

\footnotetext{
${ }^{2}$ The measures of risks for individuals and for fatalities are sometimes referred to as being utility functions for ex ante and ex post risk equity, respectively. Both Keeney and Winkler (1985) and Sarin (1985) regard the measure of social risk equity for individuals as being an index of ex ante social risk equity and the measure of social risk equity for fatalities as being the sum of an index of ex post social risk equity and an index that captures the direct concern for the number of fatalities.
} 


\section{A formal model of social risk equity evaluation}

Let $N=\{1, \ldots, n\}$ be a society consisting of $n \geq 2$ individuals, where $n \geq 2$. This society faces a social risk. The relevant outcomes for risk evaluation are the potential subsets $S \subseteq N$ of individuals who might die as a consequence of their exposure to this risk. Thus, there are $2^{n}$ mutually exclusive outcomes, from $\varnothing$ (nobody dies) to $N$ (everybody dies). Each set $S$ is a fatality set. There is uncertainty as to which of these outcomes will be realized, which is captured by a probability distribution $p$ on $2^{N}$. We refer to $p$ as a social risk distribution. Let $\mathcal{P}$ denote the set of all such probability distributions.

Following most of the literature on the evaluation of social risk equity, we restrict attention to homogeneous societies. A society is homogeneous if any differences between individuals other than their differing exposures to social risks are irrelevant for the social risk assessment. As Fishburn and Straffin (1989) and Fishburn and Sarin (1991) have noted, this assumption is not appropriate if individuals can be partitioned into homogeneous subgroups that differ from one another in a socially relevant characteristic. For example, individuals may be grouped into families or neighbourhoods, with society exhibiting a social preference for dispersing the risks across a number of these groups rather than concentrating them in a small number of them. Fishburn and Sarin (1991) have developed measures of social risk that incorporate a concern for this kind of dispersive equity.

Let $N^{*}=N \cup\{0\} . N^{*}$ is the number of individuals who might die as a result of the social risk. For all $k \in N^{*}$, let $\mathcal{T}(k)=\left\{S \in 2^{N}|| S \mid=k\right\}$ be the subsets of the population in which exactly $k$ individuals die. For all $(k, i) \in N \times N, \mathcal{S}(k, i)=\{S \in \mathcal{T}(k) \mid i \in S\} . \mathcal{S}(k, i)$ is the set of population subgroups in which person $i$ dies with $k-1$ other individuals. For the social risk distribution $p$, the probability that the outcome is a member of $\mathcal{S}(k, i)$ is $\sum_{S \in \mathcal{S}(k, i)} p(S)$.

Let $\mathcal{A}$ denote the set of probability distributions over $N$ and $\mathcal{B}$ denote the set of probability distributions over $N^{*}$. For all $p \in \mathcal{P}$, let $\alpha_{p}$ be the probability distribution in $\mathcal{A}$ whose $i$ th component is $\alpha_{p}(i)=\sum_{S \ni i} p(S)$. That is, $\alpha_{p}(i)$ is the ex ante probability that person $i$ will die and $\alpha_{p}$ is the risk profile for individuals. Similarly, for all $p \in \mathcal{P}$, let $\beta_{p}$ be the probability distribution in $\mathcal{B}$ whose $k$ th component is $\beta_{p}(k)=\sum_{S \in \mathcal{T}(k)} p(S)$. That is, $\beta_{p}(k)$ is the ex ante probability that there will be exactly $k$ fatalities and $\beta_{p}$ is the risk profile for fatalities.

A government (or other body) can undertake policies that affect the social 
risk distribution. In order to determine what policy to implement, it needs to know how to rank different social risk distributions in $\mathcal{P}$ in terms of their social acceptability. This ranking is described by a binary relation $\succeq$ on $\mathcal{P}$ which is interpreted as meaning "weakly socially preferred to." Let $\sim$ and $\succ$ denote the symmetric and asymmetric factors of $\succeq$, respectively. Henceforth, we refer to $\succeq$ as the social risk equity evaluation. Note that the restriction of $\succeq$ to social risk distributions in which there is positive probability for only one fatality set is a social preference on the set of possible ex post consequences.

There are two basic properties that we require $\succeq$ to satisfy. Our first restriction on $\succeq$ is that it is an ordering of $\mathcal{P}$.

Axiom 1 . $\succeq$ is a reflexive, complete, and transitive binary relation on $\mathcal{P}$.

We also require $\succeq$ to be continuous.

Axiom 2. The sets $\{q \in \mathcal{P} \mid p \succ q\}$ and $\{q \in \mathcal{P} \mid q \succ p\}$ are open for all $p \in \mathcal{P}$.

\section{Some examples}

As we have discussed, one approach to constructing the social risk equity evaluation $\succeq$ (or a social utility function representing this relation) is to separately construct measures of social equity for the risk profiles for individuals and for the risk profiles for the number of fatalities, and then to aggregate these measures into an overall assessment of social risk equity. With this approach, all of the relevant information about a probability distribution $p \in \mathcal{P}$ is contained in the corresponding distributions $\alpha_{p}$ and $\beta_{p}$. In effect, this approach regards social risk equity as being composed of two dimensions, with overall social risk equity being decomposable into these two components.

Such a decomposition neglects information about the distribution $p$ that may be essential for evaluating social risks, such as information abut shared destinies. It is therefore important not to assume a priori that a measure of social risk equity is decomposable. In other words, a more holistic approach is warranted, and this is provided by the social risk equity evaluation $\succeq$. Nevertheless, how society takes account of the risks that individuals face and of the possible number of fatalities plays an important role in forming an overall evaluation of social risk, even if these two dimensions of social risk do not capture all that is of social concern. The following examples illustrate the importance of these considerations.

Example 1. The set of individuals who live in some country is $N=\{1, \ldots, 100\}$. Everybody lives in either city $A$ or city $B$, both of which have the same num- 
ber of residents. For concreteness, suppose that $N_{A}=\{1, \ldots, 50\}$ live in city $A$ and $N_{B}=\{51, \ldots, 100\}$ live in city $B$. There is a probability $x$ that a hurricane will strike this country, but there is uncertainty as to its path. The hurricane will destroy city $A$ with probability $\frac{x}{2}$ and with the same probability it will destroy city $B$. In each case, all of the inhabitants of the affected city will die and all of the residents of the other city are spared.

Given its limited budget for emergency responses and the time available for hurricane preparation, the government has a choice between three options:

Option 1. Devote all of the emergency aid budget to the protection of city $A$ and the evacuation of its residents, which reduces the risks faced by residents of city $A$ to 0 , but does not change the risks faced by residents of city $B$.

Option 2. Devote all of the emergency aid budget to the protection of city $B$ and the evacuation of its residents, which reduces the risks faced by residents of city $B$ to 0 , but does not change the risks faced by residents of city $A$.

Option 3. Share equally the emergency aid budget between cities $A$ and $B$, which reduces the risk for each resident of the country to $\frac{x}{4}$.

Let $p^{j}$ be the social risk distribution corresponding to option $j, j=1,2,3$. These three distributions are summarized in the following table. Of course, $p^{j}(S)=0$ for all subgroups $S$ not shown.

\begin{tabular}{|c|c|c|c|}
\hline & $\varnothing$ & $N_{A}$ & $N_{B}$ \\
\hline \hline$p^{1}$ & $1-\frac{x}{2}$ & 0 & $\frac{x}{2}$ \\
\hline$p^{2}$ & $1-\frac{x}{2}$ & $\frac{x}{2}$ & 0 \\
\hline$p^{3}$ & $1-\frac{x}{2}$ & $\frac{x}{4}$ & $\frac{x}{4}$ \\
\hline
\end{tabular}

It can easily be checked that the risk profiles for the number of fatalities associated with these three options are as given in the following table.

\begin{tabular}{|c|c|c|c|c|c|c|c|c|}
\hline & 0 & 1 & $\cdots$ & 49 & 50 & 51 & $\cdots$ & 100 \\
\hline \hline$\beta_{p^{1}}$ & $1-\frac{x}{2}$ & 0 & $\cdots$ & 0 & $\frac{x}{2}$ & 0 & $\cdots$ & 0 \\
\hline$\beta_{p^{2}}$ & $1-\frac{x}{2}$ & 0 & $\cdots$ & 0 & $\frac{x}{2}$ & 0 & $\cdots$ & 0 \\
\hline$\beta_{p^{3}}$ & $1-\frac{x}{2}$ & 0 & $\cdots$ & 0 & $\frac{x}{2}$ & 0 & $\cdots$ & 0 \\
\hline
\end{tabular}


Thus, all three options result in the same probability distribution over the number of fatalities: with probability $1-\frac{x}{2}$, nobody will die, and with probability $\frac{x}{2}$, half the population will die. As a consequence, the expected number of deaths is the same whatever option is chosen.

The risk profiles for individuals associated with these three options are presented in the following table.

\begin{tabular}{|c|c|c|c|c|c|c|c|c|}
\hline & 1 & 2 & $\cdots$ & 50 & 51 & 52 & $\cdots$ & 100 \\
\hline \hline$\alpha_{p^{1}}$ & 0 & 0 & $\cdots$ & 0 & $\frac{x}{2}$ & $\frac{x}{2}$ & $\cdots$ & $\frac{x}{2}$ \\
\hline$\alpha_{p^{2}}$ & $\frac{x}{2}$ & $\frac{x}{2}$ & $\cdots$ & $\frac{x}{2}$ & 0 & 0 & $\cdots$ & 0 \\
\hline$\alpha_{p^{3}}$ & $\frac{x}{4}$ & $\frac{x}{4}$ & $\cdots$ & $\frac{x}{4}$ & $\frac{x}{4}$ & $\frac{x}{4}$ & $\cdots$ & $\frac{x}{4}$ \\
\hline
\end{tabular}

If options 1 or 2 are chosen, half the population has an individual probability of dying equal to $\frac{x}{2}$, whereas the other half has an individual probability of dying equal to 0 . However, if option 3 is adopted, then each individual has the same probability of dying, namely, $\frac{x}{4}$.

In this example, the three options affect how the risk of dying is distributed between individuals, but they do not differ in the probabilty that any given number of individuals will die. In effect, the government must simply decide how best to distribute the probability $\frac{x}{2}$ that half of its residents will die among all of its residents given the constraint that individuals who live in the same city must share the same destiny. On equity grounds, option 3 is socially preferred to options 1 and 2 because the individual probabilities of death are more equally distributed if option 3 is chosen. Because the society is homogeneous, it is reasonable to regard options 2 and 3 as being socially indifferent.

In general, the distribution of individual probabilities of dying is clearly not sufficient to evaluate social risk equity because it does not take into account the fairness of the resulting probabilty distribution over the number of fatalities. The following example illustrates a situation in which such considerations are decisive.

Example 2. The set of passengers on a boat is $N=\{1,2,3,4\}$. The boat is sinking, but there is only one lifeboat and it is designed for only two people. The skipper (whose code of honour requires him to go down with his boat) has the choice between the two following options:

Option 1. Let exactly half of the passengers board the lifeboat. These passengers will survive for sure, whereas the passengers who remain on 
the sinking boat will die for sure. In order to choose who boards the lifeboat, the skipper designs a fair lottery. Thus, each passenger has a probability $\frac{1}{2}$ of obtaining a place on the lifeboat.

Option 2. Let all of the passengers board the lifeboat. The overcrowded lifeboat will sink with probability $\frac{1}{2}$, leading to the death of all of the passengers. If the lifeboat does not sink, all of the passengers will survive.

Let $q^{j}$ be the social risk distribution corresponding to option $j, j=1,2$. These distributions are summarized in the following table, where $q^{j}(S)=0$ for any subgoup $S$ not shown.

\begin{tabular}{|c|c|c|c|c|c|c|c|c|}
\hline & $\varnothing$ & $\{1,2\}$ & $\{1,3\}$ & $\{1,4\}$ & $\{2,3\}$ & $\{2,4\}$ & $\{3,4\}$ & $\{1,2,3,4\}$ \\
\hline \hline$q^{1}$ & 0 & $\frac{1}{6}$ & $\frac{1}{6}$ & $\frac{1}{6}$ & $\frac{1}{6}$ & $\frac{1}{6}$ & $\frac{1}{6}$ & 0 \\
\hline$q^{2}$ & $\frac{1}{2}$ & 0 & 0 & 0 & 0 & 0 & 0 & $\frac{1}{2}$ \\
\hline
\end{tabular}

As illustrated in the following table, every individual has a probability of death equal to $\frac{1}{2}$ regardless of the option chosen.

\begin{tabular}{|c|c|c|c|c|}
\hline & 1 & 2 & 3 & 4 \\
\hline \hline$\alpha_{q^{1}}$ & $\frac{1}{2}$ & $\frac{1}{2}$ & $\frac{1}{2}$ & $\frac{1}{2}$ \\
\hline$\alpha_{q^{2}}$ & $\frac{1}{2}$ & $\frac{1}{2}$ & $\frac{1}{2}$ & $\frac{1}{2}$ \\
\hline
\end{tabular}

The probability distributions over the number of fatalities are presented in the following table.

\begin{tabular}{|l|l|l|l|l|l|}
\hline & 0 & 1 & 2 & 3 & 4 \\
\hline \hline$\beta_{q^{1}}$ & 0 & 0 & 1 & 0 & 0 \\
\hline$\beta_{q^{2}}$ & $\frac{1}{2}$ & 0 & 0 & 0 & $\frac{1}{2}$ \\
\hline
\end{tabular}

Although the expected number of deaths is the same whatever option is chosen (namely, half the number of passengers), the two options lead to very different distributions over the number of deaths. If option 1 is chosen, half the passengers will die for sure, whereas if option 2 is chosen, with probability $\frac{1}{2}$ all of the passengers will survive and with the same probability the lifeboat will sink and they all die.

In this example, it is reasonable to suppose that attitudes about the actual number of fatalities should be decisive. If one believes that society should have a preference for avoiding catastrophes, then option 1 is socially preferred 
to option 2. If one instead thinks that it is more equitable if individuals share a common fate, then option 2 is socially preferred to option 1 . However, if society expresses no concern for common fate considerations, then only the expected number of fatalities should matter in this example, with the consequence that options 1 and 2 are socially indifferent. ${ }^{3}$

In the preceding examples, nobody has to face the risk of dying alone. In Example 1, anyone who dies shares this fate with all of the other residents of the same city. In Example 2, everyone shares the same fate; the passengers either all live or they all die. Humans are social beings and we do care if our destinies are shared with others. It is the rare individual who prefers to die alone. In the following example, the risk profiles for individuals and for fatalities are identical in the two options, but some individuals face the prospect of death alone, and this is a matter of social concern.

Example 3. As in the preceding example, a boat with one lifeboat is sinking. The set of passengers on this boat is $N=\{1,2,3,4,5\}$. The lifeboat has one seat in front, two seats in the middle, and one seat in the rear. Only individuals 4 and 5 are small enough to fit in the middle seats. If four people are put in the boat, it will sink for sure if person 1 is one of them because he weighs so much. If the other four people are put in the lifeboat, with probability $\frac{1}{8}$ it will break in two because of the excessive weight. If it does break, with equal probability it will do so either just in front of or just behind the middle seats, in which case the part with three people will sink and the other part will float to safety. If there are three people including person 1 in the boat, it sinks with probability $\frac{1}{4}$. Only individuals 1,2 , and 3 know how to swim. If person 1 has to swim to shore, he has a probability of drowning of $\frac{1}{4}$. Individuals 2 and 3 are weaker swimmers, so each of them has a probability equal to $\frac{1}{8}$ of drowning if they swim. The skipper regards the following as his only two viable options:

Option 1. Let person 1 swim and put the other four people in the boat.

Option 2. Let individuals 2 and 3 swim and put the other three people in the boat.

\footnotetext{
${ }^{3}$ Keeney (1980a) has argued that society should exhibit a preference for catastrophe avoidance. Fishburn (1984) and Fishburn and Straffin (1989) have formulated axioms for social risk evaluation that distinguish between the three attitutes towards common fates described above. Bommier and Zuber (2008) have recently argued that a preference for catastrophe avoidance can provide an ethical foundation for social discounting.
} 
Let $r^{j}$ be the social risk distribution corresponding to option $j, j=1,2$. For subgroups $S$ for which the probability of dying is positive in at least one of the two options, the values of $r^{j}(S)$ are shown in the following table.

\begin{tabular}{|c|c|c|c|c|c|c|c|}
\hline & $\varnothing$ & $\{1\}$ & $\{2\}$ & $\{3\}$ & $\{1,4,5\}$ & $\{2,4,5\}$ & $\{3,4,5\}$ \\
\hline \hline$r^{1}$ & $\frac{1}{2}$ & $\frac{1}{4}$ & 0 & 0 & 0 & $\frac{1}{8}$ & $\frac{1}{8}$ \\
\hline$r^{2}$ & $\frac{1}{2}$ & 0 & $\frac{1}{8}$ & $\frac{1}{8}$ & $\frac{1}{4}$ & 0 & 0 \\
\hline
\end{tabular}

As shown in the following table, the risk profiles for these individuals are identical in the two options.

\begin{tabular}{|c|c|c|c|c|c|}
\hline & 1 & 2 & 3 & 4 & 5 \\
\hline \hline$\alpha_{r^{1}}$ & $\frac{1}{4}$ & $\frac{1}{8}$ & $\frac{1}{8}$ & $\frac{1}{4}$ & $\frac{1}{4}$ \\
\hline$\alpha_{r^{2}}$ & $\frac{1}{4}$ & $\frac{1}{8}$ & $\frac{1}{8}$ & $\frac{1}{4}$ & $\frac{1}{4}$ \\
\hline
\end{tabular}

In addition, as shown in the following table, the risk profiles for fatalities are also the same in the two options.

\begin{tabular}{|c|c|c|c|c|c|c|}
\hline & 0 & 1 & 2 & 3 & 4 & 5 \\
\hline \hline$\beta_{r^{1}}$ & $\frac{1}{2}$ & $\frac{1}{4}$ & 0 & $\frac{1}{4}$ & 0 & 0 \\
\hline$\beta_{r^{2}}$ & $\frac{1}{2}$ & $\frac{1}{4}$ & 0 & $\frac{1}{4}$ & 0 & 0 \\
\hline
\end{tabular}

If the risk profiles for individuals and for the number of fatalities were all that mattered for the evaluation of social risk equity, then the two options would be regarded as being socially indifferent. However, with $r^{1}$, individual 1 has a probability $\frac{1}{4}$ of dying alone and a probability 0 of dying with other people, whereas individuals 2 and 3 have a probability 0 of dying alone and a probability $\frac{1}{8}$ of dying with two other individuals. With $r^{2}$, individual 1 has a probability 0 of dying alone and a probability $\frac{1}{4}$ of dying with two other individuals, whereas individuals 2 and 3 have a probability $\frac{1}{8}$ of dying alone and a probability 0 of dying with other people. With both $r^{1}$ and $r^{2}$, individuals 4 and 5 each have a probability of $\frac{1}{4}$ of dying with two other individuals and a probability 0 of dying alone. One may argue that individuals feel better when they share their destiny with a larger number of people, or at least when they do not face the risk of death alone. If the social evaluation of risk equity takes these concerns into account and if, for example, society expresses a strong aversion to individuals facing the risk of dying alone, then it is reasonable to suppose that $r^{1}$ is socially preferred to $r^{2}$ because there is the same probability that someone dies alone in both options, but more people face this risk in the second option. 


\section{Shared destinies}

In this section, we propose a restriction on the social risk evaluation $\succeq$ that, when combined with Axioms 1 and 2, permits one to take account of the number of people who share one's fate, not just the risk profiles for individuals and the risk profiles for fatalities.

\subsection{Simply Related Social Risk Distributions}

Our new condition is a weakened version of an axiom introduced by Fishburn and Straffin (1989). Fishburn and Straffin were interested in identifying restrictions on the social risk evaluation that are necessary and sufficient for the overall evaluation to only depend on the risk profiles for individuals and the risk profiles for fatalities. To state Fishburn and Staffin's axiom, we first need to introduce the concept of simply related social risk distributions.

Definition 1. Two social risk distributions $p, q \in \mathcal{P}$ are simply related if either (i) $p=q$ or (ii) $p \neq q$ and there exist $A, B, C, D \subseteq N$ and $\delta>0$ such that $A \neq \varnothing, A \cap B=\varnothing,|A|=|B|,(C \cup D) \cap(A \cup B)=\varnothing, C \cup D \neq \varnothing$, and $p$ and $q$ are identical except that:
1. $q(A \cup C)=p(A \cup C)-\delta$
3. $q(A \cup D)=p(A \cup D)+\delta$
2. $q(B \cup C)=p(B \cup C)+\delta$
4. $q(B \cup D)=p(B \cup D)-\delta$.

Consider the case in which the social risk distributions $p$ and $q$ are not identical. The groups $A$ and $B$ in this definition are disjoint, but contain the same number of individuals. These two groups are also disjoint from both $C$ and $D$. The distributions $p$ and $q$ are identical except for how likely groups $A$ and $B$ are to perish with either $C$ or $D$. In the case of $A$, in moving from $p$ to $q$, some probability is shifted from dying with the members of $C$ to dying with the members of $D$. The reverse shift of the same amount of probability applies to $B$. Thus, $p$ and $q$ only differ in the relative likelihood that members of $A$ and $B$ die with members of $C$ and $D$. It is easily checked that if $p$ and $q$ are simply related, then $\left(\alpha_{p}, \beta_{p}\right)=\left(\alpha_{q}, \beta_{q}\right)$. That is, the risk profiles for individuals and the risk profiles for fatalities associated with $p$ and $q$ are identical.

For homogeneous societies, Fishburn and Straffin (1989) regard two social risk distributions as being socially indifferent if they are simply related. 
Axiom 3. For all $p, q \in \mathcal{P}, p \sim q$ if $p$ and $q$ are simply related.

Fishburn and Straffin (1989, Theorem 1) have established the following theorem.

Theorem 1. Assume that Axioms 1 and 2 hold. Then Axiom 3 holds if and only if for all $p, q \in \mathcal{P}$,

$$
\left(\alpha_{p}, \beta_{p}\right)=\left(\alpha_{q}, \beta_{q}\right) \Rightarrow p \sim q .
$$

Thus, given Axioms 1, 2, and 3, the only information that is needed to determine how to socially rank $p$ and $q$ in terms of social risk equity are the corresponding risk profiles for individuals and for fatalities. For the reasons discussed earlier, Fishburn and Straffin do not advocate the adoption of Axiom 3 in heterogeneous societies. However, they suggest that if the composition of the set of individuals who die is not of social concern, then this axiom is an appealing restriction on the social risk evaluation $\succeq$. But even if one believes that individuals should be treated symmetrically, Axiom 3 is incompatible with taking account of how many people an individual shares his fate with provided that $\succeq$ is transitive. To see why this is the case, we consider an expanded version of Example 3.

Example 4. As in Example 3, $N=\{1,2,3,4,5\}$. There are now three social risk distributions under consideration, $r^{1}, r^{2}$, and $r^{3}$. The values of $r^{j}(S)$ are shown in the following table for all the subgroups $S$ for which there is a positive probability that this subgroup perishes.

\begin{tabular}{|c|c|c|c|c|c|c|c|}
\hline & $\varnothing$ & $\{1\}$ & $\{2\}$ & $\{3\}$ & $\{1,4,5\}$ & $\{2,4,5\}$ & $\{3,4,5\}$ \\
\hline \hline$r^{1}$ & $\frac{1}{2}$ & $\frac{1}{4}$ & 0 & 0 & 0 & $\frac{1}{8}$ & $\frac{1}{8}$ \\
\hline$r^{2}$ & $\frac{1}{2}$ & 0 & $\frac{1}{8}$ & $\frac{1}{8}$ & $\frac{1}{4}$ & 0 & 0 \\
\hline$r^{3}$ & $\frac{1}{2}$ & $\frac{1}{8}$ & 0 & $\frac{1}{8}$ & $\frac{1}{8}$ & $\frac{1}{8}$ & 0 \\
\hline
\end{tabular}

Note that $r^{1}$ and $r^{2}$ are the social risk distributions considered in Example 3. The distributions $r^{1}$ and $r^{3}$ are simply related, with $A=\{1\}, B=\{3\}$, $C=\varnothing, D=\{4,5\}$, and $\delta=\frac{1}{8}$. Similarly, $r^{3}$ and $r^{2}$ are simply related, with $A=\{1\}, B=\{2\}, C=\varnothing, D=\{4,5\}$ and $\delta=\frac{1}{8}$. Therefore, Axiom 3 together with the transitivity of $\succeq$ imply that $r_{1} \sim r_{2}$. However, in our discussion of Example 3 we have argued that this preference is unacceptable if the number of people one dies with is a matter of social concern. Because the risk profiles for individuals and for fatalities are identical in all three options, the Fishburn-Straffin approach requires that they be regarded as being socially indifferent. 


\subsection{Strongly Related Social Risk Distributions}

In the definition of simply related social risk distributions, the groups $A$ and $B$ are of the same size, but $C$ and $D$ may not be. As a consequence, the redistribution of risk need not be among groups of the same size. It is for this reason that the social risk evaluation in the Fishburn-Straffin approach is insensitive to the number of individuals one dies with. To overcome this problem, we propose to weaken Axiom 3 so that it only applies to simply related social risk distributions for which the redistribution of risk is made between same-sized groups. Such distributions are called strongly related.

Definition 2. Two social risk distributions $p, q \in \mathcal{P}$ are strongly related if either (i) $p=q$ or (ii) $p \neq q$ and there exist $A, B, C, D \subseteq N$ and $\delta>0$ such that $A \neq \varnothing, A \cap B=\varnothing,|A|=|B|,|C|=|D|,(C \cup D) \cap(A \cup B)=\varnothing$, $C \cup D \neq \varnothing$, and $p$ and $q$ are identical except that:
1. $q(A \cup C)=p(A \cup C)-\delta$
3. $q(A \cup D)=p(A \cup D)+\delta$
2. $q(B \cup C)=p(B \cup C)+\delta$
4. $q(B \cup D)=p(B \cup D)-\delta$.

Clearly, if $p$ and $q$ are strongly related, then they are also simply related. For all $p, q \in \mathcal{P}$, if $p$ and $q$ are strongly related, we say that $q$ is obtained from $p$ by the strongly related shift $q-p$. We require two social risk distributions to be socially indifferent if they are strongly related. Thus, it is not a matter of social concern with whom an individual dies with, but it is of social concern how many individuals he shares this fate with.

Axiom 4. For all $p, q \in \mathcal{P}, p \sim q$ if $p$ and $q$ are strongly related.

To state our analogue of Theorem 1 , we first need to introduce some additional notation. For all $p \in \mathcal{P}$, let $M_{p}$ be the $n \times n$ matrix whose entry in the $k$ th row and $i$ th column is $M_{p}(k i)=\sum_{S \in \mathcal{S}(k, i)} p(S)$. That is, $M_{p}(k i)$ is the probability that person $i$ dies with exactly $k-1$ other individuals. Note that $\alpha_{p}(i)=\sum_{k=1}^{n} M_{p}(k i)$ for all $i \in N, \beta_{p}(k)=\frac{1}{k} \sum_{i=1}^{n} M_{p}(k i)$ for all $k \in N$, and $\beta_{p}(0)=1-\sum_{k=1}^{n} \beta_{p}(k)$. Thus, knowledge of $M_{p}$ is sufficient to compute both $\alpha_{p}$ and $\beta_{p}$.

Theorem 2. Assume that Axioms 1 and 2 hold. Then Axiom 4 holds if and only if for all $p, q \in \mathcal{P}$,

$$
M_{p}=M_{q} \Rightarrow p \sim q
$$


Proof. (a) Consider any $i \in N$. There is some group $S$ containing $i$ for which the probability of dying differs in $p$ and $q$ only if $i \in\{A, B, C, D\}$. For such $i$, because $|A|=|B|,|C|=|D|$, and $A \cup B$ is disjoint from $C \cup D$, in going from $p$ to $q$ any loss of (resp. gain in) probability for some group that $i$ is a member of is exactly compensated for by a gain in (resp. loss of) probability for some other group of the same size that also contains $i$. Hence, $M_{p}=M_{q}$. Thus, by (1), $p \sim q$.

(b) We now show that Axioms 1, 2, and 4 are sufficient for (1). For all $p \in \mathcal{P}$ and all $k \in N^{*}$ such that $\sum_{S \in \mathcal{T}(k)} p(S) \neq 0$, let $\tilde{p}^{k} \in \mathcal{P}$ be defined by setting

$$
\tilde{p}^{k}(S)=\left\{\begin{array}{cl}
\frac{p(S)}{\sum_{T \in \mathcal{T}(k)}^{p(T)}} & \text { if } S \in \mathcal{T}(k) \\
0 & \text { if } S \notin \mathcal{T}(k) .
\end{array}\right.
$$

If $\sum_{S \in \mathcal{T}(k)} p(S)=0$, let $\tilde{p}^{k}$ be defined by setting

$$
\tilde{p}^{k}(S)= \begin{cases}1 & \text { if } S=\varnothing \\ 0 & \text { if } S \neq \varnothing .\end{cases}
$$

Note that for all $p \in \mathcal{P}$ and all $(k, i) \in N \times N$,

$$
M_{p}(k i)=\left(\sum_{S \ni i} \tilde{p}^{k}(S)\right)\left(\sum_{T \in \mathcal{T}(k)} p(T)\right)=\alpha_{\tilde{p}^{k}}(i) \beta_{p}(k)
$$

and

$$
\beta_{\tilde{p}^{k}}(k)=1
$$

Furthermore, for all $p \in \mathcal{P}$,

$$
p=\sum_{k=0}^{n} \tilde{p}_{k} \beta_{p}(k)
$$

Now assume that $p, q \in \mathcal{P}$ are such that $M_{p}=M_{q}$ and, hence, that $\alpha_{p}=\alpha_{q}$ and $\beta_{p}=\beta_{q}$. Assume, furthermore, that $p(S)$ and $q(S)$ are rational for all $S$. Then, for all $(k, i) \in N \times N, \alpha_{\tilde{p}^{k}}(i)=\alpha_{\tilde{q}^{k}}(i)$. Thus, by the argument in the proof of Lemma 1 in Fishburn and Straffin (1989), for all $k \in N$, there exists a sequence $r_{1}^{k}, r_{2}^{k}, \ldots, r_{t(k)}^{k}$ of probability distributions such that $\tilde{p}^{k}$ is simply related to $r_{1}^{k}, r_{\tau}^{k}$ is simply related to $r_{\tau+1}^{k}$ for all $\tau \in\{1, \ldots, t(k)-1\}$, and $r_{t(k)}^{k}$ is simply related to $\tilde{q}^{k}{ }^{4}$ Because $\tilde{p}^{k}(S)=0$ for all $S \notin \mathcal{T}(k) \backslash\{\varnothing\}$

\footnotetext{
${ }^{4}$ Note that $\tilde{p}$ and $\tilde{q}$ have zero probability for all nonempty sets of size different from $k$. Thus, the Claim on p. 236 in Fishburn and Straffin (1989) can only hold with $|G|=k$ and $|H|=k$. This guarantees that the sets $C$ and $D$ in their proof have the same cardinality.
} 
and $\tilde{q}^{k}(S)=0$ for all $S \notin \mathcal{T}(k) \backslash\{\varnothing\}$, all of these distributions are actually strongly related. Let $s_{\tau}^{k}=r_{\tau+1}^{k}-r_{\tau}^{k}$ if $\tau \in\{1, \ldots, t(k)-1\}, s_{0}^{k}=r_{1}^{k}-\tilde{p}^{k}$, and $s_{t(k)}^{k}=\tilde{q}^{k}-r_{t(k)}^{k}$. We thus have, for all $k \in N$,

$$
\tilde{q}^{k}=\tilde{p}^{k}+\sum_{\tau=0}^{t(k)} s_{\tau}^{k} .
$$

Equations (2) and (3) imply that

$$
\begin{aligned}
q & =\sum_{k=0}^{n} \tilde{q}^{k} \beta_{q}(k) \\
& =\sum_{k=0}^{n}\left(\tilde{p}^{k}+\sum_{\tau=0}^{t(k)} s_{\tau}^{k}\right) \beta_{q}(k) \\
& =\sum_{k=0}^{n} \tilde{p}^{k} \beta_{q}(k)+\sum_{k=0}^{n} \sum_{\tau=0}^{t(k)} s_{\tau}^{k} \beta_{q}(k) \\
& =p+\sum_{k=0}^{n} \sum_{\tau=0}^{t(k)} s_{\tau}^{k} \beta_{q}(k) .
\end{aligned}
$$

Thus, $q$ is obtained by adding to $p$ a sequence of strongly related shifts. Axiom 4 therefore implies that $p \sim q$.

By using the continuity of $\succeq$ (Axiom 2), this conclusion also holds if any $p(S)$ or $q(S)$ is irrational. For the details of this extension argument, see Fishburn and Straffin (1989).

In order to illustrate the added flexibility provided by our weakening of Axiom 3, we construct a parametric family of social risk evaluation functions that are then used to evaluate the options in Example 4. Suppose that each individual $i \in N$ has a utility function $U_{i}: \mathcal{P} \rightarrow \mathbb{R}$ for which for all $p \in \mathcal{P}$,

$$
U_{i}(p)=-f\left(\sum_{S \ni i} \frac{1}{|S|^{\rho}} p(S)\right),
$$

where $f$ is increasing, strictly concave, and twice continuously differentiable, $f(0)=0$, and $\rho \in \mathbb{R}$. If $\rho=0$, then individual $i$ does not care about how many people with whom he dies. If $i \in S, S \subset S^{\prime}$, and $p(S)=p\left(S^{\prime}\right)$, then $i$ prefers to die in the larger group if and only if $\rho>0$. These observations suggest that $\rho$ is likely to be positive. 
Further suppose that the social risk evaluation $\succeq$ is represented by the evaluation function $W: \mathcal{P} \rightarrow \mathbb{R}$ given by

$$
W(p)=\sum_{i=1}^{n} f\left(U_{i}(p)\right)+V^{1}\left(\alpha_{p}\right)+V^{2}\left(\beta_{p}\right)
$$

for all $p \in \mathcal{P}$, where $V^{1}:[0,1]^{n} \rightarrow \mathbb{R}$ is a measure of social risk equity for distributions of risk for individuals and $V^{2}:[0,1]^{n+1} \rightarrow \mathbb{R}$ is a measure of social risk attitudes towards the number of fatalities.

The values of $U_{i}$ for the risk distributions $r^{1}, r^{2}$, and $r^{3}$ in Example 4 are presented in the following table.

\begin{tabular}{|c|c|c|c|c|c|}
\hline & $U_{1}$ & $U_{2}$ & $U_{3}$ & $U_{4}$ & $U_{5}$ \\
\hline \hline$r^{1}$ & $-f\left(\frac{1}{4}\right)$ & $-f\left(\frac{1}{8} \frac{1}{3^{\rho}}\right)$ & $-f\left(\frac{1}{8} \frac{1}{3 \rho}\right)$ & $-f\left(\frac{1}{4} \frac{1}{3^{\rho}}\right)$ & $-f\left(\frac{1}{4} \frac{1}{3^{\rho}}\right)$ \\
\hline$r^{2}$ & $-f\left(\frac{1}{4} \frac{1}{3^{\rho}}\right)$ & $-f\left(\frac{1}{8}\right)$ & $-f\left(\frac{1}{8}\right)$ & $-f\left(\frac{1}{4} \frac{1}{3^{\rho}}\right)$ & $-f\left(\frac{1}{4} \frac{1}{3^{\rho}}\right)$ \\
\hline$r^{3}$ & $-f\left(\frac{1}{8} \frac{1}{3^{\rho}}+\frac{1}{8}\right)$ & $-f\left(\frac{1}{8} \frac{1}{3^{\rho}}\right)$ & $-f\left(\frac{1}{8}\right)$ & $-f\left(\frac{1}{4} \frac{1}{3^{\rho}}\right)$ & $-f\left(\frac{1}{4} \frac{1}{3^{\rho}}\right)$ \\
\hline
\end{tabular}

Define the function $F: \mathcal{P} \rightarrow \mathbb{R}$ by setting

$$
F(p)=\sum_{i=1}^{3} f\left(U_{i}(p)\right)
$$

for all $p \in \mathcal{P}$. Because $U_{i}\left(r^{1}\right)=U_{i}\left(r^{2}\right)=U_{i}\left(r^{3}\right)$ for $i \in\{4,5\}, \alpha_{r^{1}}=\alpha_{r^{2}}=$ $\alpha_{r^{3}}$, and $\beta_{r^{1}}=\beta_{r^{2}}=\beta_{r^{3}}$, the three risk distributions being considered are socially ranked by the values of $F$. Letting $\theta=\frac{1}{3^{\rho}}$, these values are given by

$$
\begin{aligned}
& F\left(r^{1}\right)=-f\left(\frac{1}{4}\right)-2 f\left(\frac{\theta}{8}\right), \\
& F\left(r^{2}\right)=-f\left(\frac{\theta}{4}\right)-2 f\left(\frac{1}{8}\right),
\end{aligned}
$$

and

$$
F\left(r^{3}\right)=-f\left(\frac{1}{8}\right)-f\left(\frac{\theta}{8}\right)-f\left(\frac{\theta}{8}+\frac{1}{8}\right) .
$$

Note that $\theta=1$ when $\rho=0$ and that $\theta$ is decreasing with respect to $\rho$.

The social ranking of $r^{1}$ and $r^{2}$ can be determined from the sign of

$$
\Delta_{12}(\theta)=F\left(r^{1}\right)-F\left(r^{2}\right)=f\left(\frac{\theta}{4}\right)+2 f\left(\frac{1}{8}\right)-f\left(\frac{1}{4}\right)-2 f\left(\frac{\theta}{8}\right) .
$$


Clearly, $\Delta_{12}(1)=0$. Differentiating $\Delta_{12}(\theta)$, we obtain

$$
\frac{d \Delta_{12}(\theta)}{d \theta}=-\frac{1}{4} f^{\prime}\left(\frac{\theta}{8}\right)+\frac{1}{4} f^{\prime}\left(\frac{\theta}{4}\right)<0
$$

where the inequality follows from our assumption that $f^{\prime \prime}<0$. Therefore, $\Delta_{12}$ is increasing with respect to $\rho$. We have thus shown that $F\left(r^{1}\right)>F\left(r^{2}\right) \Leftrightarrow$ $\rho>0$; i.e., $r^{1} \succ r^{2} \Leftrightarrow \rho>0$. In our discussion of Example 3, we suggested that it is reasonable to suppose that $r^{1}$ is socially preferred to $r^{2}$ if society expresses a concern for shared destinies, which requires $\rho$ to be positive if the social risk evaluation $\succeq$ is represented by the function $W$. However, in our discussion of Example 4, we have shown that if $\succeq$ satisfies Fishburn and Straffin's three axioms, then $r^{1}$ must be socially indifferent to $r^{2}$, which is only compatible with $\rho=0 .{ }^{5}$

There are substantial informational advantages associated with constructing an index of social risk equity if two social risk distributions are judged to be socially indifferent whenever their associated distributions of risk of death for individuals and for the number of fatalities are the same. As noted by Fishburn and Straffin (1989), to characterize a social risk distribution $p \in \mathcal{P}$, $2^{n}-1$ quantities are needed, whereas only $2 n-1$ quantities are needed to compute $\alpha_{p}$ and $\beta_{p}$. The computational complexity of $M_{p}$ lies between these two extremes. For any $p \in \mathcal{P}$, the entries in the last row of the matrix $M_{p}$ are identical because everybody has the same probability of dying with $n-1$ other people. Thus, $n(n-1)+1$ quantities are needed to compute $M_{p}$. While not as informationally parsimonious as a social risk evaluation that only depends on the distributions of risks for individuals and for fatalities, our approach is less demanding than a social risk evaluation based on all of the information in a social risk distribution because the computation of $p$ is exponential in $n$, whereas the computation of $M_{p}$ is only polynomial in $n$. While such practical considerations are important, they should not be decisive if important aspects of social risk equity are neglected, such as a concern for shared destinies. For this reason, we believe that the ability to take ac-

\footnotetext{
${ }^{5}$ Similar arguments may be used to show that $r^{1} \succ r^{3} \Leftrightarrow \rho>0$ and that the social ranking of $r^{2}$ and $r^{3}$ depends on both the choice of $f$ and $\rho$. If instead of requiring $f$ to be strictly concave, we had set $f$ equal to the identity function, then for every value of $\rho$, the three risk distributions we are considering are socially indifferent. As a consequence, even though every individual expresses a concern for shared destinies when $\rho \neq 0$, society does not.
} 
count of this concern in our approach to social risk evaluation outweighs the informational advantages of the approach of Fishburn and Straffin.

\section{Concluding remarks}

We have shown that the social risk evaluation only depends on the probabilities that an individual dies with $k$ other individuals for $k=0, \ldots, n-1$ if our three axioms are satisfied. Thus, in contrast to Fishburn and Straffin (1989), the social risk evaluation is able to exhibit a concern for the shared destinies of the members of society. Our axioms are consistent with different views on how shared destinies matter. It is reasonable to believe that it is socially (and individually) preferable to perish with some other individuals rather than to perish alone, but only if the number of other individuals who die is not substantial. However, our appraoch does not commit us to this belief.

By considering additional axioms, further structure can be imposed on the social risk evaluation. A major focus of the analysis of Fishburn and Straffin (1989) is the identification of maximally consistent sets of axioms that include Axioms 1, 2, and 3. A natural extension of our analysis would be to undertake a similar excercise for Axioms 1, 2, and 4.

\section{References}

Bian, W.-Q., Keller, L. R., 1999. Chinese and Americans agree on what is fair, but disagree on what is best in societal decisions affecting health and safety risks. Risk Analysis 19, 439-452.

Bommier, A., Zuber, S., 2008. Can preferences for catastrophe avoidance reconcile social discounting with intergenerational equity? Social Choice and Welfare 31, 415-434.

Broome, J., 1982. Equity in risk bearing. Operations Research 30, 412-414.

Fishburn, P. C., 1984. Equity axioms for public risks. Operations Research 32, 901-908.

Fishburn, P. C., Sarin, R. K., 1991. Dispersive equity and social risk. Management Science 37, 751-769.

Fishburn, P. C., Straffin, P. D., 1989. Equity considerations in public risks evaluation. Operations Research 37, 229-239.

Fleurbaey, M., 2006. Assessing risky social decisions, unpublished 
manuscript, Centre de Recherche Sens, Éthique, Société, Université Paris 5 .

Harvey, C. M., 1985. Preference functions for catastrophe and risk inequity. Large Scale Systems 8, 131-146.

Keeney, R. L., 1980a. Equity and public risk. Operations Research 28, 527534.

Keeney, R. L., 1980b. Evaluating alternatives involving potential fatalities. Operations Research 28, 188-205.

Keeney, R. L., 1980c. Utility functions for equity and public risk. Management Science 26, 345-353.

Keeney, R. L., Winkler, R. L., 1985. Evaluating decision strategies for equity of public risks. Operations Research 33, 955-970.

Keller, L. R., Sarin, R. K., 1988. Equity in social risk: Some empirical observations. Risk Analysis 8, 135-146.

Sarin, R. K., 1985. Measuring equity in public risk. Operations Research 33, 210-217. 